\title{
1 Anfangen zu Schreiben
}

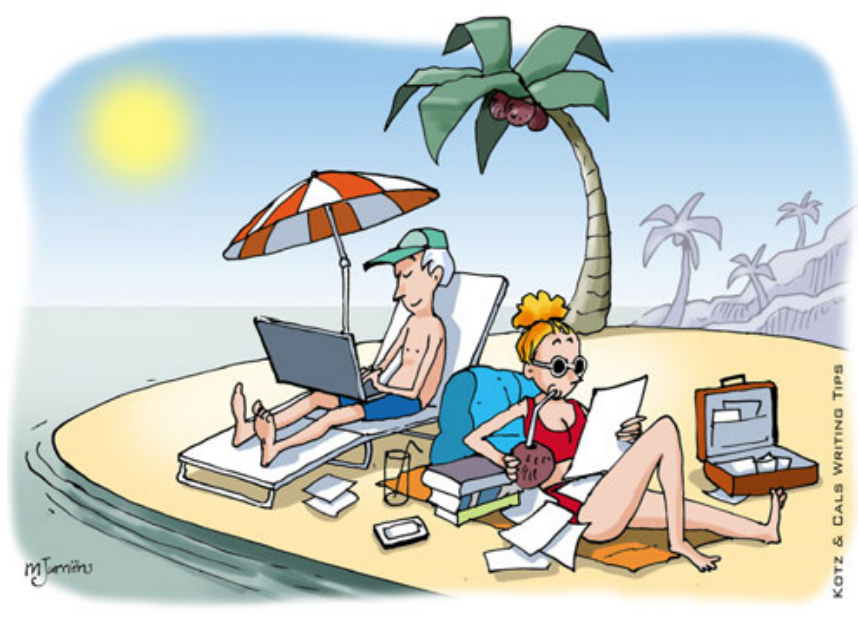

Tipp 1 - Anfangen zu Schreiben: wähle die optimale Umgebung!

\section{Was Sie wissen sollten}

Für die meisten Wissenschaftler ${ }^{\star}$ ist es schwierig, den Anfang für einen neuen Artikel zu finden und während des gesamten Schreibprozesses hindurch motiviert zu bleiben. Jeder Autor erlebt gute und schlechte Schreibtage. Es gibt jedoch eine ganze Reihe von Möglichkeiten, das Schreiben generell einfacher und angenehmer zu gestalten.

Die Reihenfolge des Schreibprozesses muss nicht unbedingt der Reihenfolge entsprechen, in der der Artikel letztendlich veröffentlicht wird, und gegebenenfalls sind einige Abschnitte einfacher zu schreiben als andere. Da die Einleitung und die abschließende Diskussion häufig als die schwierigsten Abschnitte empfunden werden, fällt es Ihnen möglicherweise leichter, mit den Methoden und den Ergebnissen anzufangen. Es kann generell vorteilhaft sein, die Einleitung und die Diskussion am Ende - aber vor der Zusammenfassung (Abstract) zu schreiben und fertigzustellen, da deren Inhalte sowohl von der gewählten Zeitschrift als auch von den in dem Artikel präsentierten Methoden und Ergebnissen abhängen.

Bevor es an das eigentliche Schreiben des Artikels geht, ist es wichtig, dass der Erstautor - also die Person, die den Schreibprozess leitet - und die an der 
Arbeit beteiligten Koautoren ein gemeinsames Verständnis des primären Forschungsziels und der zentralen Ergebnisse dieses speziellen Artikels entwickeln. Ohne eine solche Einigung wird es schwierig sein, eine klare und prägnante Abhandlung zu verfassen. Ein Artikel ist häufig nur ein Teil eines größeren Forschungsprojekts, und es gibt immer mehr über dieses Projekt zu berichten, als es die Wortbegrenzung erlaubt. Jeder individuelle Artikel muss daher sein eigenes Ziel verfolgen, und Sie müssen selbst entscheiden, was berichtet werden muss und was weggelassen werden kann. Es ist außerdem wichtig, sich möglichst frühzeitig eine passende Zeitschrift bzw. Zielgruppe festzulegen.

\section{Was Sie tun sollten}

Bevor Sie sich fragen „Wie fange ich an?“, denken Sie zunächst darüber nach, wann und wo Sie am entspanntesten, kreativsten und produktivsten schreiben. Welche Umgebung inspiriert Sie? Wo arbeiten Sie am konzentriertesten und werden am wenigsten abgelenkt? Welchen Tag in der Woche und welche Tageszeit finden Sie für das Schreiben am ertragreichsten? Es kann hilfreich sein, wenn Sie sich in Ihrem sonst vollen Tagesprogramm ein paar Blöcke von mehreren Stunden frei nehmen, in denen Sie sich explizit dem ungestörten Schreiben widmen.

Trennen Sie Denken und Schreiben! Strukturieren Sie Ihren inhaltlichen Aufbau im Kopf, bevor Sie mit dem eigentlichen Schreiben kompletter Sätze und Abschnitte beginnen. Stellen Sie wie folgt eine Gliederung auf, vor allem für die Abschnitte Einleitung und die Diskussion.

1. Verwenden Sie einzelne Worte oder Zeilen, um auf die zentrale Aussage jedes Absatzes hinzuweisen und so einen logischen und überzeugenden inhaltlichen Aufbau innerhalb des Abschnitts zu erschaffen (diese Überschriften werden später zu den „Leitsätzen“ Ihrer Abschnitte).

2. Beziehen Sie sich auf für Ihren Artikel relevante Veröffentlichungen und fügen Sie Ihren Überschriften Notizen und entsprechende Zitate hinzu.

3. Ersetzen Sie die Notizen durch grob formulierte Sätze, um daraus einen Absatz zu bilden (etwa 6-8 Sätze).

4. Überarbeiten Sie die Sätze, bis sich der Abschnitt gut liest.

5. Stellen Sie sicher, dass der Absatz über einen Einleitungssatz (d.h. einen Leitsatz, der den Inhalt des Absatzes zusammenfasst) und einen Schlusssatz (d. h. einen abschließenden Satz, der eine Brücke zum nächsten Absatz schlägt) verfügt. 
Erstellen Sie gleich zu Beginn leere Tabellen und Abbildungen. So müssen Sie gezwungenermaßen die Entscheidung treffen, welche Ergebnisse am relevantesten sind, und können dadurch einen klaren und prägnanten inhaltlichen Aufbau entwickeln. Besprechen Sie das Gerüst und die leeren Tabellen/Abbildungen mit Ihren wichtigsten Koautoren; in dieser Phase können Sie noch leicht Veränderungen vornehmen.

Bewahren Sie sich Ihre Motivation, indem Sie Schreibsitzungen in Ihren Kalender einplanen (mindestens je 2 Stunden) und die Arbeit in überschaubare Blöcke unterteilen, welche Sie jeweils in einer Sitzung erarbeiten können. Machen Sie das Schreiben während solcher Sitzungen zu Ihrer obersten Priorität und vermeiden Sie Ablenkung durch z. B. das Beantworten von E-Mails. Gehen Sie mit dem Schreibfluss mit und hören Sie auf zu schreiben, wenn Sie irgendwo steckenbleiben. Machen Sie während einer Sitzung mehrere kurze Pausen (je 5-10 Minuten), um den Kopf frei zu bekommen, und machen Sie längere Pausen (mehrere Stunden oder Tage), um über das Geschriebene zu reflektieren. Warten Sie nicht zu lange damit, um Hilfe zu bitten oder mit Koautoren über Ihren Artikel zu sprechen. Setzen Sie sich realistische Zwischenziele (z. B. „Ich werde meinen Koautoren bis zum Ende der Woche die Gliederung meiner Einleitung präsentieren“) und legen Sie Ihr Endziel fest (z. B. „Ich werde meinen Artikel vor dem Ende des Monats bei einer Zeitschrift einreichen“). Zu guter Letzt: Belohnen Sie sich selbst, wenn Sie Ihre Zwischenziele oder Ihr Endziel erreicht haben!

\section{Checkliste für den Einstieg in das Schreiben einer Arbeit}

- Legen Sie Zeiten zum Schreiben fest und wählen Sie eine optimale Umgebung

- Trennen Sie Denken und Schreiben: Strukturieren Sie Ihren kompletten inhaltlichen Aufbau und erstellen Sie leere Tabellen/Abbildungen, bevor Sie mit dem eigentlichen Schreiben ganzer Sätze oder Abschnitte beginnen

- Entscheiden Sie sich schon früh für eine mögliche Zeitschrift

- Verteilen Sie den Schreibprozess auf überschaubare Blöcke

- Schaffen Sie Schreibsitzungen und machen Sie ausreichend kurze und lange Pausen

- Belohnen Sie sich selbst für das Erreichen Ihrer Zwischen- und Endziele 